5. Trân Diệu Linh, Lê Anh Tuấn, and Phan Thị Thu Nga (2013). Nghiên cứu tình hình bệnh lý và tử vong tré sơ sinh non tháng - thấp cân tại Bệnh viện Phụ sản Trung ương năm 2010. Tạp chí P̉hụ sản, 11(2), 65-69.

6. Aghai Z.H., Goudar S.S., Patel A., et al. (2020). Gender variations in neonatal and early infant mortality in India and Pakistan: a secondary analysis from the Global Network Maternal Newborn Health Registry. Reprod Health, 17(3), 178.
7. Yasmin S., Osrin D., Paul E., et al. (2001). Neonatal mortality of low-birth-weight infants in Bangladesh. Bull World Health Organ, 79(7), 608-614.

8. Roberts D., Brown J., Medley N., et al. (2017). Antenatal corticosteroids for accelerating fetal lung maturation for women at risk of preterm birth. Cochrane Database Syst Rev, 3, CD004454.

9. Lee H.C. and Gould J.B. (2006). Survival rates and mode of delivery for vertex preterm neonates according to small- or appropriate-for-gestationalage status. Pediatrics, 118(6), e1836-1844.

\title{
XÂY DỰNG MÔ HÌNH PHÂN TÍCH TÁC ĐộNG NGÂN SÁCH RUXOLITINIB TRONG ĐIỀU TRỊ XO' TỦY NGUYỀN PHÁT TẠI VIỆT NAM
}

\section{TÓM TẮT}

Xơ tủy nguyên phát (XTNP) là bênh lý huyết học được tiên lượng điều trị bất lợi nhất trong nhóm bệnh tăng sinh tủy ác tính. Thuốc ruxolitinib (RUX) được chứng minh làm giúp giảm thể tích lách và nguy cơ tử vong so với tri liêu tốt nhất hiên có (Best available therapy - BAT) trong điêu trị XTNP. Với giá thành thuốc cao, cần thiết phải xem xét tính khả thi khi lựa chọn thuốc trong thực hành lâm sàng dựa trên phẩn tích tác động ngân sách (TĐNS), trong đó xây dựng mô hình đánh giá TĐNS được xem là một trong những bước quan trọng quyết định tính khả thi và độ tin cậy của nghiên cứu. Với phương pháp mô hình hóa kết hợp với tổng quan tài liệu và tham vấn ý kiến các chuyên lâm sàng, nghiên cứu đã xây dựng được mô hình đánh giá TĐNS RUX trong điều trị XTNP tại Việt Nam trên nền tảng phần mềm MS Microsoft Excel 2013 với cấu trúc mô hình bao gồm các trang tính toán cho các Tham số đâu ra (tác đônng ngân sách và hiệu quả) cho 2 viễn cảnh không có RUX và có RUX. Mổ hình được xây dựng cho phép phân tích tác động ngân sách của RUX với các mức thanh toán bảo hiểm y tế khác nhau trong điều trị xơ tủy nguyên phát cũng như dư báo được tác động trong hiệu quả điều trị giữa hai viê̂n cảnh nghiên cứu.

Tư khóa: Phân tích tác động ngân sách, ruxolitinib, xơ tủy nguyên phát, mô hình.

\section{SUMMARY \\ CONSTRUCT THE MODEL OF BUDGET IMPACT ANALYSIS OF RUXOLITINIB IN PRIMARY MYELOFIBROSIS IN VIETNAM}

Primary myelofibrosis (PMF) is the hematologic disease which has the most unfavorable prognosis in

*Đai hoc Y Dược Thành phố Hồ Chí Minh Chịu trách nhiệm chính: Nguyễn Thị Thu Thủy Email: nguyenthuthuy@ump.edu.vn Ngày nhận bài: 7.6.2021

Ngày phản biện khoa học: 2.8.2021

Ngày duyệt bài: 12.8.2021
Nguyễn Gia Bảo*, Nguyễn Thị Huệ*, Hoàng Kim Trúc*, Nguyễn Thị Thu Thủy*

the group of myeloproliferative neoplasms diseases. Ruxolitinib (RUX) has been shown to significantly reduce spleen volume and risk of death compared with the best available therapy (BAT) in PMF treatment. With high drug costs, it is necessary to consider the feasibility of drug selection in clinical practice based on budget impact analysis (BIA), in which constructing model is considered one of the most important steps, deciding the feasibility and reliability of BIA. With modeling research method combined with literature review and indepth-interview with clinical experts, the BIA model has been built based on MS Microsoft Excel 2013 software with model structure including calculation pages for outcome parameters (budget impact and effectiveness impact) for 2 scenarios without RUX and with RUX. The model allows to conduct BIA of RUX with different health insurance payments in PMF as well as evaluate the effectiveness impact of treatment between the two research scenarios.

Keywords: Budget impact analysis, ruxolitinib, primary myelofibrosis, model

\section{I. ĐĂT VẤN ĐỀ}

Xơ tủy nguyên phát (XTNP) là bệnh lý huyết học được tiên lượng điều trị bất lợi nhất trong nhóm bệnh tăng sinh tủy ác tính ${ }^{1}$. Do đó, chí phí điều trị XTNP đang ngày càng trở thành gánh nặng. RUX là chất ức chế JAK1/JAK2 được chứng minh làm giảm thể tích lách và nguy cơ tử vong so với trị liệu tốt nhất hiện có (BAT - Best available therapy) thông qua nghiên cứu COMFORT- II trong điêu trị XTNP2,3. Với giá thành thuốc cao, cân thiết phải xem xét tính khả thi khi lựa chọn thuốc trong thực hành lâm sàng dựa trên phân tích tác động ngân sách (TĐNS). Măc dù nhiều nghiên cứu phân tích TĐNS đã được tiến hành trên thế giới ${ }^{4}$ tuy nhiên chưa có nghiên cứu được tiến hành tại Việt Nam. Hơn thế nữa, để thực hiện phân tích TĐ̣S một trong những công cụ quan trọng là xây dựng mô hình 
phù hợp với bối cảnh điều trị tại Việt Nam. Vì vậy, nghiên cứu được tiến hành nhằm mục tiêu xâyy dựng mô hình để phản ánh thực trạng điều trị xơ tủy tại Việt Nam tạo công cụ phân tích TĐNS của RUX trong điều trị XTNP.

\section{II. ĐỐI TƯợNG VÀ PHƯƠNG PHÁP NGHIÊN CỨU}

2.1. Đối tượng nghiên cứu. Mô hình phân tích tác động ngân sách của RUX trong điều trị XTNP

\subsection{Phương pháp nghiên cứu}

Phương pháp: Mô hình hóa kết hợp tổng quan tài liệu và tham vấn ý kiến chuyên gia

Các bước xây dựng mô hình tác động ngân sách được trình bày trong hình 1.

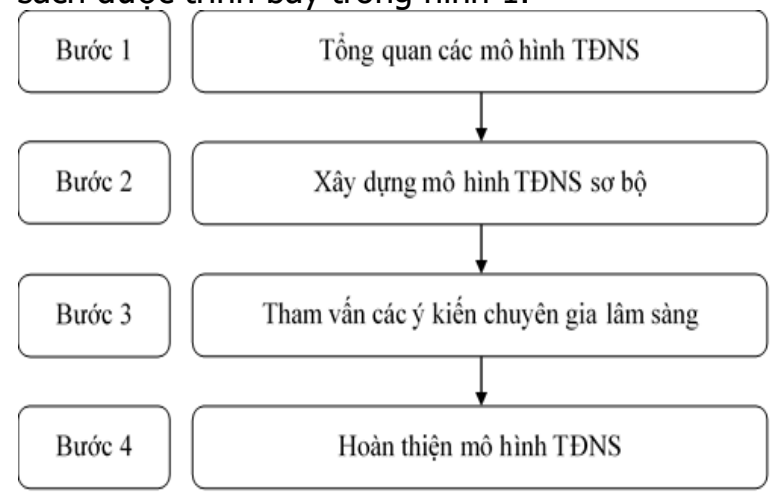

Hình 1. Các bước xây dựng mô hình

Theo hình 1, mô hình tác động ngân sách được xây dựng thông qua bốn bước bao gồm tổng quan y văn, xây dựng mô hình sơ bộ, tham vấn ý kiến chuyên gia lâm sàng và hoàn thiện mô hình. Trong đó dựa trên tổng quan các y văn, đề tài xem xét đặc điểm các mô hình phân tích TĐNS của RUX trong điều trị xơ tủy nguyên phát đã được công bố, từ đó xây dựng mô hình sơ bô. Mô hình sơ bộ được hiệu chỉnh bởi các chuyền gia lâm sàng thông qua tham vấn ý kiến chuyên gia, nhờ đó mô hình được hoàn thiện phù hợp với bối cảnh lâm sàng tại Việt Nam.

Bước 1: Tổng quan các mô hình phân tích TĐNS được thực hiện bằng phương pháp tổng quan hệ thống theo hướng dẫn PRISMA

Tìm kiếm các nghiên cứu: Nghiên cứu tiến hành tìm kiếm trên các nguồn dữ liệu thư viện điện tử bao gồm Pubmed, Cochrane, Embase, Science Direct và Google Scholar với câu lệnh tìm kiếm dựa trên các từ khóa: "Budget Impact Analysis", "primary myelofibrosis" và toán tử AND.

Sàng lọc và lựa chọn nghiên cứu: Các nghiên cứu tìm thấy được lựa chọn thông qua các tiêu chí lựa chọn (nghiên cứu phân tích TĐNS của RUX trong điều trị XTNP, nghiên cứu được viết bằng tiếng $A n h$ ) và loại trừ (nghiên cứu không có bài toàn văn, tổng quan hệ thống, thư gửi ban biên tập, báo cáo ca, bình luận).

Trích xuất, tống hợp và trình bày dữ liệu

Các nghiên cứu đáp ứng tiêu chí lựa chọn và loại trừ được trích xuất thông tin bao gồm đặc điểm nghiên cứu (tên tác giả, quốc gia, năm nghiên cứu, phân tích kinh tế), thiết kế mô hình (đặc điểm mô hình, chức năng mô hình).

Bước 2: Xây dựng mô hình sơ bộ

Đề tài xây dựng mô hình sơ bộ phân tích TĐNS trong điều trị xơ tủy nguyên phát với các đặc điểm thỏa mãn các tiêu chís được trình bày trong bảng 1 .

Bảng 1. Những yêu cầu của mô hình sơ bộ

\begin{tabular}{|c|c|}
\hline Đặc điểm & Yêu câu \\
\hline $\begin{array}{c}\text { Quẩn thể } \\
\text { nghiên cứu }\end{array}$ & $\begin{array}{c}\text { Bao gôm tất cả những người } \\
\text { bệnh XTNP và đủ điều kiện sử } \\
\text { dụng thuốc RUX }\end{array}$ \\
\hline $\begin{array}{c}\text { Viển cảnh } \\
\text { so sánh }\end{array}$ & $\begin{array}{c}\text { Phản ánh các can thiệp điều trị } \\
\text { hiện tại và tương lai }\end{array}$ \\
\hline $\begin{array}{c}\text { Khung thời } \\
\text { gian } \\
\text { nghiên cứu }\end{array}$ & $\begin{array}{c}\text { Phù hớp với khuyến cáo và } \\
\text { hướng dẫn BIA hiện tại }\end{array}$ \\
\hline $\begin{array}{c}\text { Quan điếm } \\
\text { nghiên cứu }\end{array}$ & $\begin{array}{c}\text { Phù hợp với đối tượng sử dụng } \\
\text { kết quả nghiển cứu }\end{array}$ \\
\hline
\end{tabular}

Bước 3. Tham vấn ý kiến chuyên gia. Vớ nội dung tham vấn bao gồm các yêu câu và đặc điểm của mô hình, đề tài tiến hành tham vấn 10 chuyên gia lâm sàng tại các bênh viện Truyền máu - Huyết học Thành phố Hồ Chí Minh, Viên Huyết học - Truyền máu Trung ương, Chợ Rấy, Bach Mai được lựa chọn dựa trên tiêu chí lựa chọn và tiêu chí loại trừ được trình bày trong bảng 3 .

\section{Tiêu chí lựa chọn}

- Bác sĩ cơ hữu

- Chuyên khoa huyết học hoặc ung thư

- Có kinh nghiệm điều trị trên 10 năm

- Đồng ý tham gia nghiên cứu

Tiêu chí loại trừ

Không cung cấp đầy đủ các thông tin cho buổi phỏng vấn

Bước 4. Xây dựng mô hình hoàn thiên

Sau khi tham vẩn ý kiến chuyên gia, mổ hình sẽ được hoàn thiện các trang tính toán, trang Tham số đầu vào đầu ra để trở thành công cụ phân tích TĐNS.

\section{KẾT QUẢ NGHIÊN CỨU}

3.1. Tổng quan các mô hình phân tích tác động ngân sách. Dựa trên cơ sở dữ liệu và câu lệnh nghiên cứu, đề tài tìm thây 98 nghiên cứu từ các cơ sở dữ liệu tìm kiếm. Sau khi loại bỏ trùng lặp, tiến hành lựa chọn và loại trừ dựa trên các tiêu chí đã đề ra, đề tài thu được 1 nghiên 
cứu để đưa vào phân tích. Kết quả được trình bày trong hình 2.

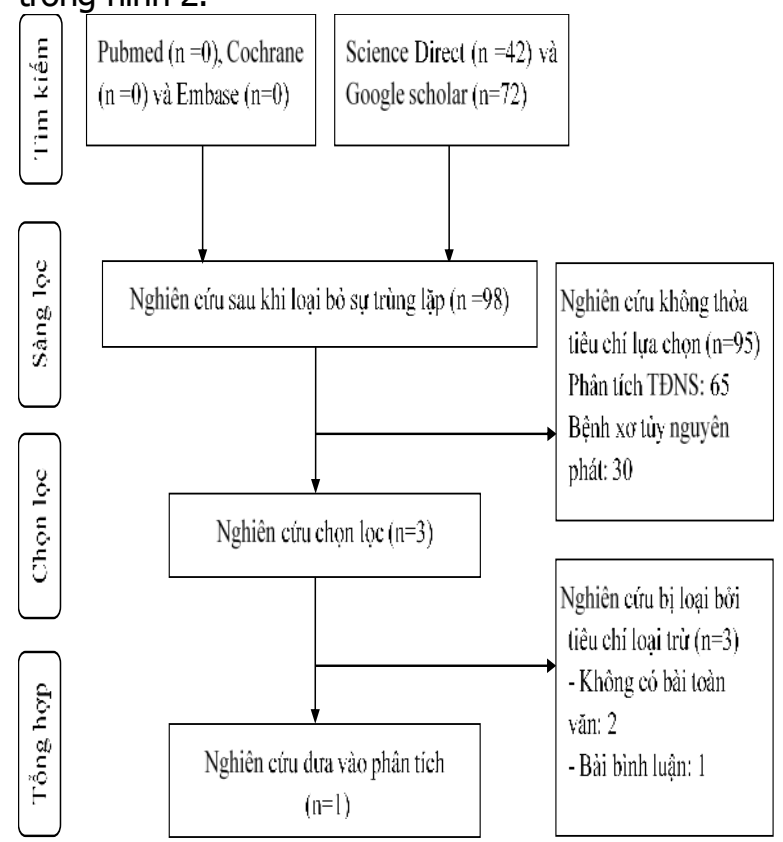

Hình 2. Lưu đồ PRISMA

Theo hình 2, nghiên cứu phân tích tác động ngân sách của RUX trong điều trị xơ tủy nguyên phát tại Ailen (2015) của tác giả Hickey $\mathrm{N}$ và cộng sựt . Nghiên cứu này sử dụng mô hình động để phân tích tác động ngân sách. Cấu trúc của mô hình gồm hai viễn cảnh điều trị có RUX và không có RUX. Dân số mục tiêu bao gồm người bệnh điều trị xơ tủy và phẩn loại tiên lượng nguy cơ trung bình-2 hoăc cao theo phân loại IPSS. Để theo dõi diễn tiến bệnh xơ tủy tác giả đã xây dựng mô hình Markov và được trình bày trong hình 3 .

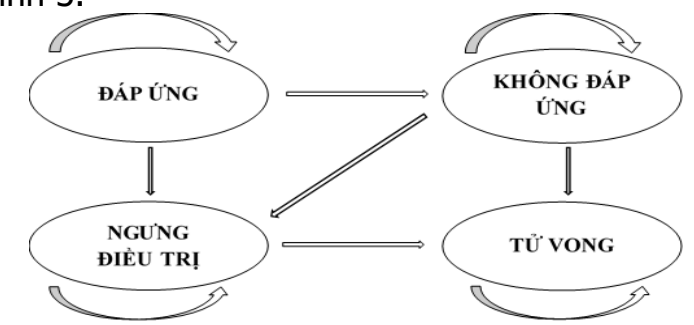

\section{Hình 3. Mô hình Makov theo dối diến tiên người bệnh xơ tủy tai Ailen}

Theo hình 3 , mô hình Markov Hickey $\mathrm{N}$ và cộng sự bao gồm bốn trạng thái (đáp ứng, không đáp ứng, ngưng điều trị và tử vong) với chu kỳ mô hình Markov 12 tuần, tần số chuyển của mô hình được rút ra từ nghiên cứu lâm sàng COMFORT-II.

3.2. Mô hình sơ bộ phân tích tác động ngân sách
Cấu trúc của mô hình. Dựa trên tổng quan các mô hình và các yêu cầu xây dựng mô hình phân tích TĐNS, đề tài xây dựng mô hình phân tích TĐNS sơ bộ với cấu trúc được trình bày trong hình 4.

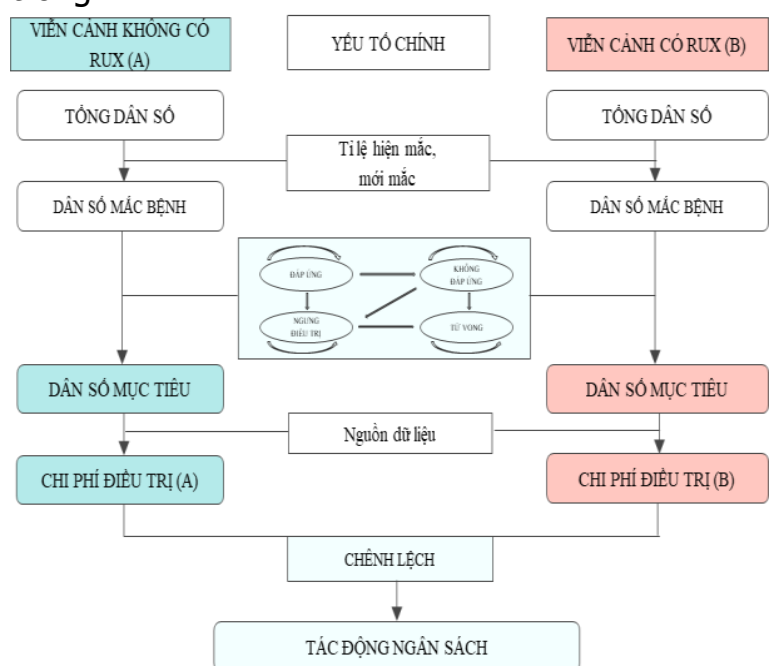

Hình 4. Cấu trúc của mô hình phân tích TĐNS

Theo hình 4, mô hình được xây dựng trong phần mềm MS Microsoft Excel 2013 với cấu trúc mô hình bao gồm các trang tính toán các Tham số đầu vào của mô hình (dân số mục tiêu, chi phí điều trị) cho 2 viễn cảnh không có RUX và có RUX. Mô hình phân tích TĐNS có các đăc điểm được trình bày trong bảng 4 .

Bảng 4. Đặc điểm của mô hinh sơ bộ

\begin{tabular}{|c|c|}
\hline Đăcc điểm & Yêu câuu \\
\hline $\begin{array}{c}\text { Quẩn thể nghiên } \\
\text { cứu }\end{array}$ & $\begin{array}{c}\text { Quần thể động được ước lượng } \\
\text { dựa trên dân số mắc bệnh và } \\
\text { mố hình Markov theo dỗi quẩn } \\
\text { thể mắc bệnh trong thời gian } \\
\text { nghiến cứu TĐNS }\end{array}$ \\
\hline $\begin{array}{c}\text { Viễn cảnh so } \\
\text { sánh }\end{array}$ & $\begin{array}{c}\text { Viễn cảnh không có RUX: bao } \\
\text { gồm BAT } \\
\text { Viễn cảnh có RUX: bao gồm } \\
\text { RUX và BAT }\end{array}$ \\
\hline $\begin{array}{l}\text { Khung thời gian } \\
\text { nghiên cứu }\end{array}$ & 5 năm \\
\hline $\begin{array}{l}\text { Quan điểm } \\
\text { nghiên cứu }\end{array}$ & Cơ quan chi trả BHYT Việt Nam \\
\hline
\end{tabular}

Giả định của mô hình: Người bệnh có đặc điểm dịch tễ, tỷ lệ biến chứng và tác dụng phụ tương đương với dân số nghiên cứu COMFORT-II.

\section{Chức năng của mô hình}

- Ước lượng và phân tích được chi phí điều trị qua các năm ở các phương án điều trị nghiên cứu.

- Đánh giá được tác động của RUX trong điều trị xơ tủy nguyên phát với các phương án điều tri khác nhau. 
- Cho phép cập nhật các Tham số đầu vào của mô hình để thu được kết quả phản ánh thực tế chính xác nhất.

Bảng 4. Nội dung cần hiệu chỉnh

\begin{tabular}{|c|c|c|}
\hline \\
\hline Nội dung & Đặc điểm & Ý kiến của các chuyên gia \\
\hline \multicolumn{3}{|c|}{ Các yêu câu của mô hình } \\
\hline 1. Quần thể nghiên cứu & $\begin{array}{l}\text { Quần thể động được ước lượng dựa } \\
\text { trên dân số mắc bệnh và mô hình } \\
\text { Markov theo dõi quẩn thể mắc bệnh } \\
\text { trong thời gian nghiên cứu TĐNS }\end{array}$ & $\begin{array}{c}\text { Cân tính đến sự thay đổi quy } \\
\text { mô quần thể nghiên cứu phụ } \\
\text { thuộc vào thanh toán BHYT } \\
\text { của RUX }\end{array}$ \\
\hline 2. Viễn cảnh điều trị & $\begin{array}{l}\text { Viển cảnh không có RUX: bao gồm } \\
\text { BAT } \\
\text { Viễn cảnh có RUX: bao gồm RUX và } \\
\text { BAT }\end{array}$ & $\begin{array}{c}\text { Tỷ lệ sử dưng các phác đồ } \\
\text { tính đến khả năng chi trả của } \\
\text { người bệnh }\end{array}$ \\
\hline $\begin{array}{l}\text { 3. Khung thời gian nghiên } \\
\text { cứu }\end{array}$ & 5 năm & $\begin{array}{l}\text { Thiết kế linh động để có thể } \\
\text { thay đổi khung thời gian } \\
\text { nghiên cứu 3-5 năm }\end{array}$ \\
\hline 4. Quan điểm nghiên cứu & Cơ quan chi trả BHYT & Phù hợp \\
\hline \multicolumn{3}{|c|}{ Đặc điểm của mô hình } \\
\hline 1.Cấu trúc của mô hình & $\begin{array}{l}\text { Bao gôm các trang tính toán trong } \\
\text { MS Excel } 2013\end{array}$ & Phù hợp \\
\hline 2.Giả định của mô hình & $\begin{array}{l}\text { Người bệnh có đặc điểm dịch tể, tỷ lệ } \\
\text { tác dụng phụ, biến chứng tương } \\
\text { đương nghiền cứu COMFORT II }\end{array}$ & $\begin{array}{l}\text { Bổ sung một số giả định khác } \\
\text { liên quan đến điêuu trị }\end{array}$ \\
\hline 3.Chức năng của mô hình & $\begin{array}{l}\text { - Ước lượng và phân tích được chi phí } \\
\text { điều trị qua các năm ớ các phương } \\
\text { án điều trị nghiên cứu. } \\
\text { - Đánh giá được tác động của RUX } \\
\text { trong điêuu trị xơ tủy nguyên phát với } \\
\text { các phương án điêu trị khác nhau } \\
\text { - Cho phép cập nhật các Tham số } \\
\text { đâuu vào của mồ hình để thu được kết } \\
\text { quả phản ánh thực tế chính xác nhất. }\end{array}$ & $\begin{array}{l}\text { Bổ sung chức năng đánh giá } \\
\text { đước tác động ở khía cạnh } \\
\text { hiệuu quả trên toàn dân số } \\
\text { thô̂ng qua số ca cứu sống }\end{array}$ \\
\hline 4. Mô hình Markov & $\begin{array}{c}\text { Bốn trang thái: đáp ứng, không đáp } \\
\text { ứng, ngưng điêuu trị, tử vong }\end{array}$ & $\begin{array}{c}\text { Mô hình nên hiệu chỉnh thành } \\
\text { ba trạng thái ổn định, tiến } \\
\text { triển và tử vong }\end{array}$ \\
\hline $\begin{array}{l}\text { 5. Chu kỳ mô hình } \\
\text { Markov }\end{array}$ & 12 tuần & $\begin{array}{l}\text { Chu kỳ của mô hình hiệu } \\
\text { chỉnh } 4 \text { tuần ( } 1 \text { tháng) }\end{array}$ \\
\hline
\end{tabular}

3.4. Mô hình đánh giá TĐNS hoàn thiên

Tham số đâu vào và đâu ra của mô hình. Các tham số đầu vào và đầu ra của mô hình đánh giá TĐNS được trình bày trong bảng 5 .

Bảng 5. Các tham số đầu ra và đầu vào của mô hình

\begin{tabular}{|c|c|c|}
\hline STT & Các tham số của mô hình & Nội dung \\
\hline \multicolumn{3}{|r|}{ Tham số đâu vào } \\
\hline 1 & Tân số chuyển & $\begin{array}{c}\text { Tần số chuyển từ trang thái ổn định sang tiến triển và từ } \\
\text { tiến triển sang tử vong ở viễn cảnh }\end{array}$ \\
\hline 2 & Dân số mục tiêu & Bao gồm dân số hiện mắc và mới mắc \\
\hline 3 & Chi phí điều trị & $\begin{array}{l}\text { Bao gồm chi phí hai trang thái: } \\
\text { - Chi phí trạng thái ổn định: thuốc, tác dụng phụ, quản lý } \\
\text { bệnh, truyền máu } \\
\text { - Chi phí trạng thái tiến triển: biến chứng lách to, bệnh } \\
\text { bạch cầu cấp tính, chăm sóc giảm nhẹ }\end{array}$ \\
\hline
\end{tabular}

3.3 Tham vấn ý kiến chuyên gia. Dựa trên tham vấn ý kiến nhóm chuyên gia, đề tài ghi nhận những hiệu chỉnh cần được thực hiện với kết quả trình bày trong bảng 4 . 


\begin{tabular}{|c|c|c|}
\hline 4 & Tỷ lệ sử dụng & $\begin{array}{c}\text { Tỷ lệ sử dụng phác đồ BAT và RUX ở hiện tại và tương lai } \\
\text { trong } 2 \text { viễn cảnh có và không có RUX }\end{array}$ \\
\hline \multicolumn{3}{|r|}{ Tham số đâu ra } \\
\hline 1 & Kết quả tác động ngân sách & Sự chênh lệch ngân sách giữa hai viê̂n cảnh \\
\hline 2 & Kết quả tác động hiệu quả & Sự chênh lệch số ca cứu sống giữa hai viền cảnh \\
\hline 3 & Phân tích độ nhạy & Mức độ ảnh hưởng của các yếu tố đến mô hình TĐNS \\
\hline
\end{tabular}

Cấu trúc mô hình. Mô hình xây dựng trên hai viễn cảnh điều trị hiện tai và tương lai với kết quả được trình bày trong hình 5 .

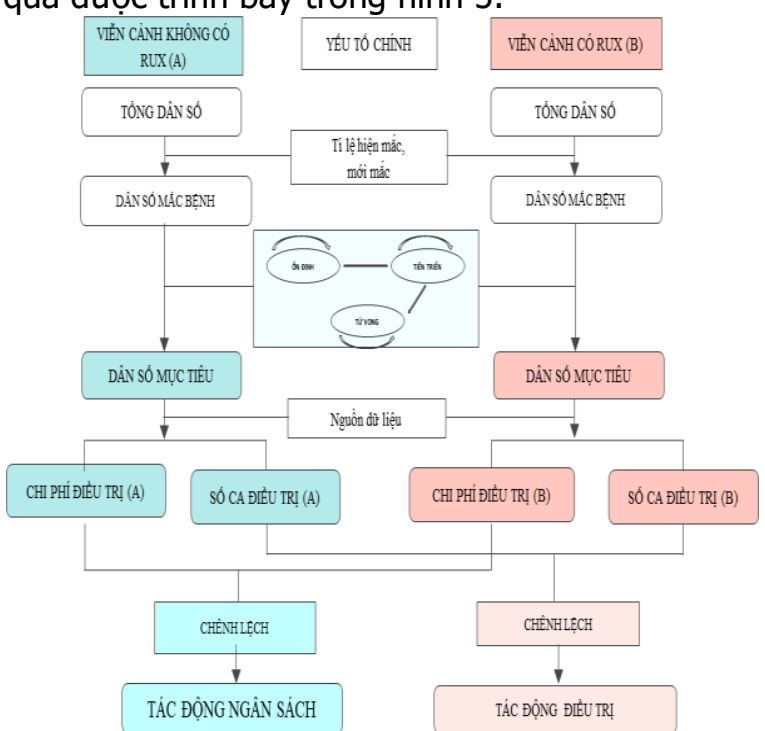

Hình 5. Cấu trúc mô hình hoàn thiện

Theo hình 5, sau khi tham vấn ý kiến chuyên gia, mô hình phân tích TĐNS được hoàn thiện với cấu trúc bao gồm các trang tính toán được xây dựng bằng phần mềm MS Microsoft Excel 2013. Trong đó:

- Dân số mục tiêu được đánh giá dựa trên dữ liệu dịch tễ và mô hình Markov bao gồm 3 trạng thái (ổn định, tiến triển, tử vong).

- Tổng tác động ngân sách và tác đông hiệu quả được ghi nhận bằng mức chênh lệch chi phí và số ca cứu sống giữa hai viễn cảnh

\section{Giả đinh của mô hình}

- Ngưới bệnh có đặc điểm dịch tễ, tỷ lê biến chứng và tác dụng phụ tương đương với dân số nghiên cứu của nghiên cứu lâm sàng COMFORT-II.

- Tần số chuyển giữa các trạng thái ổn định và không thay đổi

- Người bệnh tuân thủ điều trị.

\section{Chức năng của mô hình}

- Ước lượng số lượng người bệnh ở mỗi trạng thái sau mỗi chu kì Markov, từ đó tính toán được chi phí và hiệu quả của từng phác đồ.

- Ước lượng được cấu trúc và so sánh được ngân sách điều trị qua các năm ở các phương án điều trị nghiên cứu.
- Đánh giá sự thay đổi các tham số đầu ra khi thay đổi các tham số đầu vào.

\section{BÀN LUÂ̂N}

Nghiên cứu đã tiến hành tìm kiếm dữ liệu bằng phương pháp tổng quan hệ thống với cẩu lệnh và từ khóa chặt chẽ trên các nguồn dữ liêu đáng tin cậy bao gồm Pubmed, thư viện Cochrane, Science direct, Embase và Google scholar. Kết quả tìm kiếm cho thây chỉ 1 nghiên cứu thỏa tiêu chí được lựa chọn để tổng hợp. Giới hạn về số lượng nghiển cứu thỏa tiêu chí có thể được giải thích bởi số lượng nghiên cứu công bố về phân tích tác động ngẩn sách của RUX vẫn còn hạn chế do bệnh lý xớ tủy là bệnh hiếm và RUX là can thiệp mới. Hơn nữa việc lựa chọn ngôn ngữ tiếng Anh có thể giới hạn những nghiên cứu bằng ngôn ngữ khác.

Dựa trên tổng quan mô hình, các yêu cầu xây dựng mô hình và tham vấn các chuyên gia, nghiên cứu đã xây dựng mô hình động nhằm phân tích tác động ngân sách của RUX trong điều trị xớ tủy. Cấu trúc của mô hình gồm hai viễn cảnh có và không có RUX. Khung thời gian nghiên cứu được xem xét là 5 năm, điều này phù hợp với thực trạng của cơ quan quản lý nhằm giúp cho các nhà hoạch định chính sách có cái nhìn tổng quan và đủ dài để ra các quyết định đúng đắn. So sánh với nghiên cứu của Hickey và cộng sự", mô hình hoàn thiện bổ sung kết quả cho những tỷ lệ hoàn trả RUX khác nhau, bổ sung tác động hiệu quả bên cạnh TĐNS.

Để theo dõi diễn tiến người bệnh xơ tủy tại Việt Nam, mô hình Markov bao gồm ba trạng thái ổn định, tiến triển, tử vong và chu kỳ Markov 1 tháng được thiết lập. Điêu này tương đồng với nghiên cứu tại Tây Ban Nha về chi phí hiệu quả của RUX trong điều trị PMF'.

Sử dụng hợp lý các phương pháp phân tích kinh tế dược, mô hình xây dựng có thể cung cấp công cụ hữu ích cho cơ quan quản lý y tế trong việc xem xét TĐNS của RUX trong điều trị bệnh xớ tủy nguyên phát tại Việt Nam. Mô hình được xây dựng để đánh giá TĐNS của RUX trong điểu trị PMF tại Việt Nam vì vậy khi áp dụng trên các can thiệp khác ở cùng bệnh lý, cần phải có những điêu chỉnh phù hợp. 


\section{KẾT LUẬN}

Mô hình được xây dựng. Mô hình được xây dựng trong phần mềm MS Microsoft Excel với cẩu trúc mô hình bao gồm các trang tính toán các tham số đầu ra cho 2 viễn cảnh không có RUX và có RUX cho phép phân tích tác động ngân sách của RUX trong điều trị xơ tủy nguyên phát, đánh giá được tác động hiệu quả điều trị giữa hai viễn cảnh điều trị và cho phép cập nhật các tham số đầu vào đề thu được kết quả cập nhật nhất cho từng thời điểm nghiên cứu nhất định.

\section{TÀI LIÊU THAM KHẢO}

1. Tefferi A. Primary myelofibrosis: 2021 update on diagnosis, risk-stratification and management. American journal of hematology. 2021;96(1):145-162.

2. Harrison $C N$, Vannucchi AM, Kiladjian J-J, et al. Long-term findings from COMFORT-II, a phase
3 study of ruxolitinib vs best available therapy for myelofibrosis. Leukemia. 2016;30(8):1701-1707.

3. Cervantes F, Vannucchi AM, Kiladjian JJ, et al. Three-year efficacy, safety, and survival findings from COMFORT-II, a phase 3 study comparing ruxolitinib with best available therapy for myelofibrosis. Blood. 2013;122(25):4047-4053.

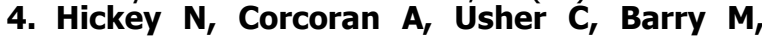
McCullagh L. 2017; A462. Available at. Accessed 9, 20.

5. Marshall DA, Douglas PR, Drummond MF, et al. Guidelines for conducting pharmaceutical budget impact analyses for submission to public drug plans in Canada. Pharmacoeconomics. 2008;26(6):477-495.

6. Gómez-Casares MT, Hernández-Boluda JC, Jiménez-Velasco A, et al. Cost-effectiveness of Ruxolitinib vs Best Available Therapy in the Treatment of Myelofibrosis in Spain. Journal of Health Economics and Outcomes Research. 2017;5(2):162-174.

\section{CHI PHÍ ĐIỀU TRI TRỰC TIẾP BÊ̂NH LOẠN SẢN PHẾ QUẢN PHỔI TẠI BỆNH VIỆN NHI TRUNG ƯƠNG NĂM 2020}

\section{TÓM TẮT}

Muc tiêu: Mô tả chi phí điều tri trực tiếp cho y tế và các yếu tố liên quan trong điều trị trẻ sơ sinh mắc chứng loạn sản phế quản phổi tại Bệnh viện Nhi Trung ương trong năm 2020. Phương pháp nghiên cứu: Mô tả cắt ngang, phân tích định lượng dữ liệu hồi cứu của tất cả trẻ sơ sinh điều trị chứng loạn sản phế quản phổi tại Bệnh viện trong 2020. Kết quả: Trong tổng 116 đối tượng nghiên cứu, nam là chủ yếu với 63,8\%, tỷ lệ trẻ đẻ non <28 tuân là $59,8 \%$. Tổng chi phí trực tiếp cho y tế trung bình năm/1 trẻ là 3292,5 $\pm 3043,8$ USD. Chi phí này tỷ lệ thuận với cân nặng khi sinh và thời gian nằm viện. Kết luẩn: Chi phí ở Việt Nam thấp hơn đáng kể so với chi phí quốc tế ở các nghiên cứu tương tự. Chính sách bảo hiểm y tế miến phí cho trẻ < 6 tuổi và tỷ lệ đồng chi trả < $20 \%$ làm giảm đáng kể gánh năng kinh tế của gia đình có trẻ phải điều trị chứng lìạn sản phế quản phổi và hoàn toàn nằm trong khả năng chi trả.

Tư khóa: Chi phí, chứng loạn sản phế quản phổi, trẻ sơ sinh, Bệnh viện Nhi Trung ương

\section{SUMMARY}

${ }^{1}$ Bệnh viện Nhi Trung ương

2Trường Đại học Y Hà Nội

${ }^{3}$ Vu Kế hoạch Tài chính - Bô Y tế

Chịu trách nhiệm chính: Trần Thị Thu Trang

Email: tranthithutrang33@gmail.com

Ngày nhận bài: 2.6.2021

Ngày phản biện khoa học: 2.8.2021

Ngày duyệt bài: 11.8.2021
Trần Thị Thu Trang ${ }^{1}$, Trần Minh Điển ${ }^{1}$, Phạm Huy Tuấn Kiệt ${ }^{2}$, Vũ Xuân Hoàng ${ }^{3}$

\section{DIRECT TREATMENT COST OF BRONCHOPULMONARY DYSPLASIA AT VIETNAM NATIONAL CHILDREN'S HOSPITAL IN 2020}

Objectives: To describe direct medical cost and related factors for treatment of bronchopulmonary dysplasia (BPD) at the Vietnam National Children's Hospital in 2020. Methodology: Cross-sectional study, quantitative analysis on medical and financial records of all premature infants with bronchopulmonary dysplasia, treated at the National Children's Hospital in 2020. Findings: Among 116 premature infants with bronchopulmonary dysplasia, males in majority with $63,8 \%$, premature $<28$ weeks with $59,8 \%$. The average annual total direct medical cost per infant was $3292.5 \pm 3043.8$ USD. Value of cost is proportional to birth weight and length of stay. Conclusion: Cost in Vietnam is significantly comparing with similar international studies. Policy of free health insurance for children $<6$ year olds and co-payment of $<20 \%$ reduced the financial burden of families having premature infant with bronchopulmonary dysplasia largely. It was totally affordable in Vietnam.

Keywords: Cost, bronchopulmonary dysplasia, infants, Vietnam National Children's Hospital

\section{I. ĐĂT VẤN ĐỀ}

Theo Tổ chức $Y$ tế Thế giới (WHO), cứ mỗi năm lại có 15 triệu trẻ sinh non toàn cầu. Đây được cho là nguyền nhân tử vong hàng đầu ở trẻ em dưới 5 tuổi ở các nước có thu nhập thấp và trung bình [1]. Bệnh lý lâm sàng phổ biến nhất ở 